\title{
LIMIT CYCLES FOR A CLASS OF CONTINUOUS PIECEWISE LINEAR DIFFERENTIAL SYSTEMS WITH THREE ZONES
}

\author{
MAURíCIO FIRMINO SILVA LIMA \\ Centro de Matemática Computação e Cognição. Universidade Federal do \\ ABC, 09210-170. Santo André. S.P. Brazil, e-mail: \\ mauricio.lima@ufabc.edu.br \\ JAUME LLIBRE \\ Departament de Matemàtiques \\ Universitat Autònoma de Barcelona, 08193. Bellaterra, Barcelona, Spain, \\ e-mail: jllibre@mat.uab.es
}

\begin{abstract}
In this paper we consider a class of planar continuous piecewise linear vector fields with three zones. Using the Poincaré map we show that these systems admit always a unique limit cycle, which is hyperbolic.
\end{abstract}

\section{Introduction}

Due to the encouraging increase in their applications, control theory [Lefschetz, 1965] and [Narendra et al., 1973], design of electric circuits [Chua et al., 1990], neurobiology [FitzHugh, 1961] and [Nagumo et al., 1962] piecewise linear differential systems were studied early from the point of view of qualitative theory of ordinary differential equations [Andronov et al., 1966]. Nowadays, a lot of papers are being devoted to these differential systems.

On the other hand, starting from linear theory, in order to capture nonlinear phenomena, a natural step is to consider piecewise linear systems. As local linearizations are widely used to study local behavior, global linearizations (achieved quite naturally by working with models which are piecewise linear) can help to understand the richness of complex phenomena observed in the nonlinear world.
The study of piecewise linear systems can be a difficult task that is not within the scope of traditional nonlinear systems analysis techniques. In particular, a sound bifurcation theory is lacking for such systems due to their nonsmooth character.

In this paper we study the existence of limit cycles for the class of continuous piecewise linear differential systems

$$
\mathbf{x}^{\prime}=X(\mathbf{x}),
$$

where $\mathbf{x}=(x, y) \in \mathbb{R}^{2}$, and $X$ is a continuous piecewise linear vector field. We will consider the following situation, that we will name the three-zone case. We have two parallel straight lines $L_{-}$and $L_{+}$symmetric with respect to the origin dividing the phase plane in three closed regions: $R_{-}, R_{o}$ and $R_{+}$with $(0,0) \in R_{o}$ and the regions $R_{-}$and $R_{+}$have as boundary the straight lines $L_{-}$and $L_{+}$ respectively. We will denote by $X_{-}$the vector field $X$ restrict to $R_{-}$, by $X_{o}$ the vector field $X$ restricted 
to $R_{o}$ and by $X_{+}$the vector field $X$ restrict to $R_{+}$. We suppose that the restriction of the vector field to each one of these zones are linear systems with constant coefficients that are glued continuously at the common boundary.

In short, system (1) can be written as

$$
\mathbf{x}^{\prime}= \begin{cases}A_{-} \mathbf{x}+B_{-} & \mathbf{x} \in R_{-} \\ A_{o} \mathbf{x}+B_{o} & \mathbf{x} \in R_{o} \\ A_{+} \mathbf{x}+B_{+} & \mathbf{x} \in R_{+}\end{cases}
$$

where $A_{i} \in \mathcal{M}_{2}(\mathbb{R}), i \in\{-, o,+\}, B_{i} \in \mathbb{R}^{2}, i \in$ $\{-, o,+\}$ and $\mathbf{x}^{\prime}=\frac{d \mathbf{x}}{d t}$ with $t$ the time.

We say that the vector field $X$ has a real equilibrium $x^{*}$ in $R_{i}$ with $i \in\{-, o,+\}$ if $x^{*}$ is an equilibrium of $X_{i}$ and $x^{*} \in R_{i}$. In opposite we will say that $X$ has a virtual equilibrium $x^{*}$ in $R_{i}$ if $x^{*} \in R_{i}^{c}$ where $R_{i}^{c}$ denotes the complementary of $R_{i}$ in $\mathbb{R}^{2}$.

We suppose the following assumptions:

(H1) $X_{o}$ has a real equilibrium in the interior of the region $R_{o}$ of focus type.

(H2) The others equilibria (real or virtual) of $X_{-}$ and $X_{+}$are a center and a focus with different stability with respect to the focus of $X_{O}$.

We note that if the two equilibria of $X_{-}$and $X_{+}$are both centers, or a center and a focus having this focus the same stability than the focus of $X_{O}$, then the vector field $X$ has no limit cycles, for more details see Proposition 2.4.

As usual a limit cycle of (2) is a periodic orbit of (2) isolated in the set of all periodic orbits of (2). A limit cycle is hyperbolic if the integral of the divergent of the system along it is different from zero, for more details see for instance [Dumortier et al., 2006].

Our main result is the following.

Theorem 1.1. Assume that system (2) satisfies assumptions (H1) and (H2). Then system (2) has a unique limit cycle, which is hyperbolic .

More information about the limit cycle of Theorem 1.1 is given in Propositions 4.1 and 4.2, where it is characterized when the limit cycle visit two or three zones.

We observe that Theorem 1.1 is in some situations an extension of Proposition 15 of [Freire et al., 1998]. This proposition shows that a piecewise linear differential system with two zones can have at most one limit cycle. More precisely we have that the limit cycle stated in Proposition $15(\mathrm{e})$ is persistent in the present scenario where we have three zones having a center in $R_{-}$or in $R_{+}$.

\section{Normal Form}

The aim of this section is to write system (2) in a convenient normal form where the number of parameters are reduced, and consequently the computations of the Poincaré return map will be easier.

Lemma 2.1. Under the assumptions (H1) and (H2) there exists a linear change of coordinates that writes system (2) into the form

$$
\dot{\mathbf{x}}=X(\mathbf{x}),
$$

with $L_{-}=L_{-1}=\{x=-1\}, L_{+}=L_{1}=\{x=$ $1\}, R_{-}=\left\{(x, y) \in \mathbb{R}^{2} ; x \leq-1\right\}, R_{o}=\{(x, y) \in$ $\left.\mathbb{R}^{2} ;-1 \leq x \leq 1\right\}, R_{+}=\left\{(x, y) \in \mathbb{R}^{2} ; x \geq 1\right\}$ and

$$
X(\mathbf{x})= \begin{cases}A_{-} \mathbf{x}+B_{-} & \mathbf{x} \in R_{-} \\ A_{o} \mathbf{x}+B_{o} & \mathbf{x} \in R_{o} \\ A_{+} \mathbf{x}+B_{+} & \mathbf{x} \in R_{+}\end{cases}
$$

where $A_{-}=\left(\begin{array}{cc}a_{11} & -1 \\ 1-b_{2}+d_{2} & a_{1}\end{array}\right), B_{-}=$ $\left(\begin{array}{c}a_{11} \\ d_{2}\end{array}\right), A_{o}=\left(\begin{array}{cc}0 & -1 \\ 1 & a_{1}\end{array}\right), B_{o}=\left(\begin{array}{c}0 \\ b_{2}\end{array}\right), A_{+}=$ $\left(\begin{array}{cc}c_{11} & -1 \\ 1+b_{2}-f_{2} & a_{1}\end{array}\right)$ and $B_{+}=\left(\begin{array}{c}-c_{11} \\ f_{2}\end{array}\right)$. The dot denotes derivative with respect to a new time $s$.

Proof. Under the hypotheses, by means of a rotation and a homothecy in the $x$ direction we can write the system in such a way that $L_{-}=L_{-1}$, $L_{+}=L_{1}$. Now doing the change of coordinates given by $u=x, v=k_{1} x+k_{2} y+k_{3}$ and the time rescaling $t=k_{4} s$ with

$$
\begin{gathered}
k_{1}=-\frac{b_{11}}{\sqrt{b_{11} b_{22}-b_{12} b_{21}}}, k_{2}=-\frac{b_{12}}{\sqrt{b_{11} b_{22}-b_{12} b_{21}}}, \\
k_{3}=-\frac{e_{1}}{\sqrt{b_{11} b_{22}-b_{12} b_{21}}}, k_{4}=\frac{1}{\sqrt{b_{11} b_{22}-b_{12} b_{21}}},
\end{gathered}
$$

where $b_{i j}, i, j=1,2$ are the initial entries of the matrix $A_{o}$ and $B_{o}=\left(e_{1}, e_{2}\right)$. Note that $X_{O}$ in these coordinates becomes $X_{o}\left(\begin{array}{l}u \\ v\end{array}\right)=$ 
$\left(\begin{array}{cc}0 & -1 \\ 1 & a_{1}\end{array}\right)\left(\begin{array}{l}u \\ v\end{array}\right)+\left(\begin{array}{c}0 \\ b_{2}\end{array}\right)$, and it has equilibrium in $x_{o}^{*}=\left(-b_{2}, 0\right)$ where $\left|b_{2}\right|<1$.

We observe that the straight lines $L_{-}$and $L_{+}$are invariant under the change of coordinate $(x, y, t) \rightarrow(u, v, s)$.

The expressions of the vector fields $X_{-}$and $X_{+}$ follows by the continuity in $L_{-}$and $L_{+}$respectively.

For our purpose we will define a first return map that involves all the vector fields $X_{-}, X_{O}$ and $X_{+}$in a suitable transversal section. In the case considered in this paper this section will be a segment of the line $L_{-}$and the next remark and lemma state conditions for the existence of a such map.

Remark 2.2. In [Llibre et al., 2004] and [Llibre et al., 2009] Theorem 4.3 .10 we see that a necessary and sufficient condition for the existence of Poincaré maps from the straight lines $L_{ \pm}$to the straight lines $L_{ \pm}$is that there exists a unique contact point of the flow of the linear system with these lines. By contact point we mean a point of the line where the vector field is tangent to it.

Lemma 2.3. In the coordinates given by Lemma 2.1 there is a unique contact point of system (3) with $L_{-}$and a unique contact point of (3) with $L_{+}$. These points are respectively $p_{-}=(-1,0)$ and $p_{+}=$ $(1,0)$. Moreover the equilibria of $X_{-}$and $X_{+}$are virtual.

Proof. The proof of the first part of this lemma follows easily by direct computations. For the second part note that the eigenvalues of $A_{-}$are given by the expression $\left[\left(a_{11}+a_{1}\right) \pm\right.$ $\left.\sqrt{\left(a_{11}+a_{1}\right)^{2}-4\left(a_{11} a_{1}+1-b_{2}+d_{2}\right)}\right] / 2$ and the eigenvalues of $A_{+}$are given by the expression [ $\left(c_{11}+\right.$ $\left.\left.a_{1}\right) \pm \sqrt{\left(c_{11}+a_{1}\right)^{2}-4\left(c_{11} c_{1}+1+b_{2}-f_{2}\right)}\right] / 2$. So in order that the equilibrium points of $X_{-}$and $X_{+}$ be of center or focus type we must have $a_{11} a_{1}+1-$ $b_{2}+d_{2}>0$ and $c_{11} c_{1}+1+b_{2}-f_{2}>0$.

Now the equilibrium of $X_{-}$is given by $x_{-}^{*}=\left(-\frac{a_{1} a_{11}+d_{2}}{a_{1} a_{11}+1-b_{2}+d_{2}},-\frac{-a_{11}+a_{11} b_{2}}{a_{1} a_{11}+1-b_{2}+d_{2}}\right)$ with $\left|b_{2}\right|<1$ and from the fact that $a_{11} a_{1}+1-b_{2}+d_{2}>0$ we can see by direct computation that $-\frac{a_{1} a_{11}+d_{2}}{a_{1} a_{11}+1-b_{2}+d_{2}}>-1$. This implies that $x_{-}^{*}$ is a virtual equilibrium of the system. The same argument is valid for the equilibrium $x_{+}^{*}$ of $X_{+}$.

In the rest of the paper for $i \in\{-, o,+\}$ we denote $t_{i}$ the trace of matrix $A_{i}$, and by $d_{i}$ as been the determinant of the matrix $A_{i}$.

Denote by $\operatorname{Int}(\Gamma)$ the open region limited by the closed Jordan curve $\Gamma$.

The next result is an immediate consequence of the Green's Formula (see for instance Proposition 3 of [Llibre et al., 1996]).

Proposition 2.4. If system (3) has a simple invariant closed curve $\Gamma$ then

$$
\iint_{\operatorname{In} t_{-}(\Gamma)} t_{-} d x d y+\iint_{\operatorname{Int} t_{o}(\Gamma)} t_{o} d x d y+
$$

$$
\iint_{\operatorname{Int}_{+}(\Gamma)} t_{+} d x d y=t_{-} S_{-}+t_{o} S_{o}+t_{+} S_{+}=0
$$

where $\operatorname{Int}_{i}(\Gamma)=\operatorname{Int}(\Gamma) \cap R_{i}$ and $S_{i}=$ area $\left(\operatorname{Int}_{i}(\Gamma)\right)$ with $i \in\{-, o,+\}$.

Observe that if we have a focus in $R_{o}$ and a center in $R_{-}$or $R_{+}$then a necessary condition for the existence of such $\Gamma$ is that $\Gamma$ visit at least the two zones having a focus and that the stability of both foci is different.

\section{Poincaré Return Map}

We have that system (3) has a unique real equilibrium in $R_{o}$ and, by Lemma 2.3, the two other equilibria are virtual.

In order to study the existence of limit cycles for system (3) we will define a Poincaré return map defined on $L_{-}$. This Poincaré return map will be defined as the composition of four different Poincaré maps. In what follows we study the qualitative behavior of each one of these maps separately in order to understand the global behavior of the general Poincaré return map.

The next lemma will be useful in the study of the Poincaré return map associated to system (3). The proof of this lemma can be found in [Llibre et al., 2009] Lemma 4.4.10 but in order to have a complete understood of the results we will reproduce the proof here. 
Lemma 3.1. Consider the function $\varphi: \mathbb{R}^{2} \rightarrow \mathbb{R}$ given by $\varphi(x, y)=1-e^{x y}(\cos x-y \sin x)$. The qualitative behavior of $\varphi\left(x, y_{0}\right)$ in $(-\infty, 2 \pi)$ is represented in Figure 1 when $y_{0}>0$, and Figure 2 when $y_{o}<0$.

Proof. Since $\frac{\partial \varphi}{\partial x}=\left(1+y^{2}\right) e^{x y} \sin x$ the critical value of $\varphi$ are $x_{k}=k \pi$, where $k \in \mathbb{Z}$. From $\frac{\partial^{2} \varphi}{\partial x^{2}}=(1+$ $\left.y^{2}\right) e^{x y}(\cos x+y \sin x)$ and assuming that $y_{o}>0$ it follows that $\varphi$ has a local minimum at $x_{k}$ for $k$ even or has a local maximum at $x_{k}$ for $k$ odd.

On the other hand when $p \in \mathbb{Z}$ and $p<0$ we have $\varphi\left(x_{2 p}, y_{o}\right)=1-e^{2 p \pi y_{o}}>0$, and consequently $\varphi\left(x, y_{o}\right)>0$ when $x<0$. Moreover $\varphi\left(0, y_{0}\right)=0$, $\varphi\left(\pi, y_{o}\right)=1+e^{\pi y_{o}}>0$ and $\varphi\left(2 \pi, y_{o}\right)=1-e^{2 \pi y_{o}}<$

0 . Therefore there exists a unique zero $\tau$ in $(0,2 \pi)$ which proves the lemma for $y_{0}>0$. The case $y_{0}<0$ follows by applying that $\varphi(-x, y)=\varphi(x,-y)$.

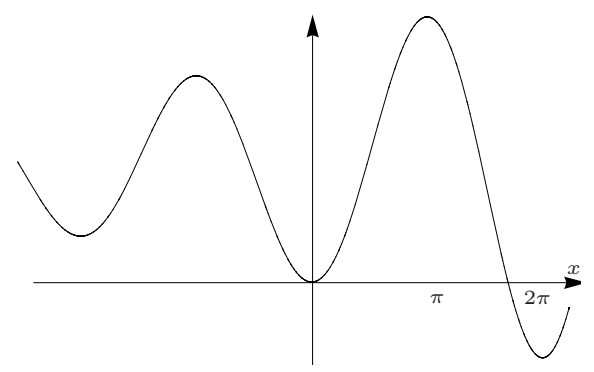

Fig. 1. Function $\varphi\left(x, y_{o}\right)$ for $y_{o}>0$.

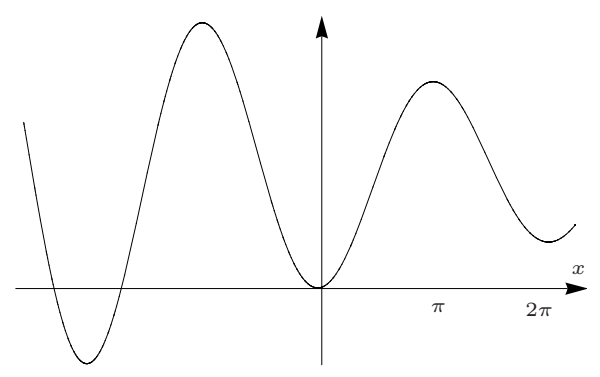

Fig. 2. Function $\varphi\left(x, y_{o}\right)$ for $y_{o}<0$.

Let $p_{-}$be the contact point of $\dot{\mathbf{x}}=A_{-} \mathbf{x}+B_{-}$ with $L_{-}$. Note that $p_{-}$divides $L_{-}$into two segments $L_{-}^{O}$ and $L_{-}^{I}$ where in $L_{-}^{O}$ the vector field points toward the region $R_{-}$while in $L_{-}^{I}$ the vector field points toward the region $R_{O}$. In fact we have $L_{-}^{O}=\{(-1, y) ; y \geq 0\}$ and $L_{-}^{I}=\{(-1, y) ; y \leq 0\}$.
We can define a Poincaré map $\Pi_{-}: L_{-}^{O} \rightarrow L_{-}^{I}$ by $\Pi_{-}(p)=q$ as been the first return map in forward time of the flow of $\dot{\mathbf{x}}=A_{-} \mathbf{x}+B_{-}$to $L_{-}$, that is, if $\varphi_{-}(s, p)$ is the solution of $\dot{\mathbf{x}}=A_{-} \mathbf{x}+B_{-}$ such that $\varphi_{-}(0, p)=p$ and $p \in L_{-}^{O}$, then $q=$ $\varphi_{-}(s, p), s \geq 0$ such that $q \in L_{-}^{I}$. Observe that $\Pi_{-}\left(p_{-}\right)=p_{-}$.

We can see the mapping $\Pi_{-}$in a different way as follows. Given, $p \in L_{-}^{O}$ and $q \in L_{-}^{I}$ there exist unique $a \geq 0$ and $b \geq 0$ such that $p=p_{-}-a \dot{p}_{-}$ where $\dot{p}_{-}=X_{-}\left(p_{-}\right)=\left(0, b_{2}-1\right)$, and $q=p_{-}+b \dot{p}_{-}$. So the mapping $\Pi_{-}$induces a mapping $\pi_{-}$given by $\pi_{-}(a)=b$.

Note that to study the qualitative behavior of $\Pi_{-}$is equivalent to study the qualitative behavior of $\pi_{-}$. From now on we will consider the map $\pi_{-}$ instead of $\Pi_{-}$.

From Proposition 4.3.7 in [Llibre et al., 2009] the mapping $\pi_{-}$previously defined is invariant under change of coordinates and translation for which the equilibrium $x_{-}^{*}$ remains virtual. So for computing the mapping $\pi_{-}$we can suppose that the virtual equilibrium is at the origin and that the matrix $A_{-}$ is given in its real Jordan normal form.

Note that we can define in the same way a map $\pi_{+}$associated to the a Poincaré map $\Pi_{+}$in $L_{+}$considering the flow defined by $\dot{\mathbf{x}}=A_{+} \mathbf{x}+B_{+}$and the contact point $p_{+}$.

Proposition 3.2. Consider the vector field $X_{i}$ in $R_{i}$ with $i \in\{-,+\}$, with a virtual center or focus equilibrium and such that $t_{i} \geq 0$. Let $\pi_{i}$ be the map associated to the Poincaré map $\Pi_{i}: L_{i} \rightarrow L_{i}$ defined by the flow of the linear system $\dot{\mathbf{x}}=A_{i} \mathbf{x}+B_{i}$.

(a) If $t_{i}>0$ then the maps $\pi_{i}$ satisfy that $\pi_{i}$ : $[0, \infty) \rightarrow[0, \infty), \pi_{i}(0)=0, \lim _{a \rightarrow \infty} \pi_{i}(a)=+\infty$ and $\pi_{i}(a)>a$ in $(0, \infty)$.

(a.1) If $a \in(0, \infty)$ then $\left(\pi_{i}\right)^{\prime}(a)=\frac{a}{\pi_{i}(a)} e^{2 \gamma_{i} \tau_{i}}$. Moreover $\left(\pi_{i}\right)^{\prime}(a)>1$ and $\lim _{a \rightarrow 0}\left(\pi_{i}\right)^{\prime}(a)=$ 1.

(a.2) If $a \in(0, \infty)$ then $\left(\pi_{i}\right)^{\prime \prime}(a)>0$.

(a.3) The straight line $b=e^{\gamma_{i} \pi} a-t_{i}(1+$ $\left.e^{\gamma_{i} \pi}\right) / d_{i}$ in the plane $(a, b)$ is an asymptote of the graph of $\pi_{i}$ when a tends to $+\infty$ where $\gamma_{i}=t_{i} / \sqrt{4 d_{i}-t_{i}^{2}}$. So $\lim _{a \rightarrow \infty}\left(\pi_{1}\right)^{\prime}(a)=e^{\gamma_{i} \pi}$. 
(b) It $t_{i}=0$ then $\pi_{i}$ is the identity in $[0, \infty)$.

Proof. We will prove the result for $i=-$. The case $i=+$ follows in the same way.

Let $p_{-}$be the contact point of the flow with $L_{-}$ and $p$ and $q$ as described above. As $q$ is in the orbit of $p$ in the forward time we have that $q=\varphi(s, p)$ with $s \geq 0$. Moreover for computing the map $\pi_{-}$ we can suppose that the virtual equilibrium is at the origin and that matrix $A_{-}$is in its real Jordan normal form.

Let $p_{-}^{*}$ be the contact point $p_{-}$in the coordinates in which $A_{-}$is in its real Jordan normal form and the virtual equilibrium of $X_{-}$is at the origin. We denote by $\dot{p}_{-}^{*}=X_{-}\left(p_{-}^{*}\right)$. So we can write

$$
q=\varphi(s, p)=e^{A_{-} s} p .
$$

As $p=p_{-}^{*}-a \dot{p}_{-}^{*}$ and $q=p_{-}^{*}+\pi_{-}(a) \dot{p}_{-}^{*}$ we obtain

$$
p_{-}^{*}+\pi_{-}(a) \dot{p}_{-}^{*}=e^{A_{-} s}\left(p_{-}^{*}-a \dot{p}_{-}^{*}\right) .
$$

Now using the fact that $\dot{p}_{-}^{*}=A_{-} p_{1}^{*}$ we have

$$
\left(I d+\pi_{-}(a) A_{-}\right) p_{-}^{*}=e^{A_{-} s}\left(I d-a A_{-}\right) p_{-}^{*},
$$

where $a \geq 0, \pi_{-}(a) \geq 0, s \geq 0$ and the matrix $A_{-}$ is given by $A_{-}=\left(\begin{array}{cc}\alpha_{-} & -\beta_{-} \\ \beta_{-} & \alpha_{-}\end{array}\right)$with $\alpha_{-}=\frac{t_{-}}{2}$. Since $p_{-}^{*} \neq(0,0)$ we obtain from equation (4) that $b=\pi_{-}(a)$ is defined by the system

$1+b \alpha_{-}=e^{\alpha_{-} s}\left(\cos \left(\beta_{-} s\right)+a\left[\beta_{-} \sin \left(\beta_{-} s\right)-\alpha_{-} \cos \left(\beta_{-} s\right)\right]\right)$,

$b \beta_{-}=e^{\alpha_{-} s}\left(\sin \left(\beta_{-} s\right)-a\left[\alpha_{-} \sin \left(\beta_{-} s\right)+\beta_{-} \cos \left(\beta_{-} s\right)\right]\right)$,

and the inequalities $a \geq 0, b \geq 0$ and $s \geq 0$.

Note that $s=0, a=0$ and $b=0$ is a solution of system (5). So we have $\pi_{-}(0)=0$. Moreover if $a=a_{o}, b=b_{o}$ and $s=s_{o}$ is a solution of (5), then $s_{o}$ is the flight time between the points $p=p_{-}^{*}-a \dot{p}_{-}^{*}$ and $q=p_{-}^{*}+b \dot{p}_{-}^{*}$. Thus $\beta_{-} s$ is the angle between $p$ and $q$ and consequently $\beta_{-} s \in[0, \pi)$.

Define $\tau_{-}=\beta_{-} s$ and $\gamma_{-}=\alpha_{-} / \beta_{-}$. Solving system (5) with respect to $\tau_{-} \in(0, \pi)$ we obtain the following parametric equations for $\pi_{-}(a)=b$,

$$
\begin{aligned}
a\left(\tau_{-}\right) & =\frac{\beta_{-} e^{-\gamma_{-} \tau_{-}}}{d_{1} \sin \tau_{-}} \varphi\left(\tau_{-}, \gamma_{-}\right) \quad \text { and } \\
b\left(\tau_{-}\right) & =\frac{\beta_{-} e^{\gamma_{-} \tau_{-}}}{d_{1} \sin \tau_{-}} \varphi\left(\tau_{-},-\gamma_{-}\right),
\end{aligned}
$$

where $\varphi$ is the function described in Lemma 3.1.

Now since $\lim _{\tau_{-} \rightarrow \pi} a\left(\tau_{-}\right)=\infty$ and $\lim _{\tau_{-} \rightarrow \pi} b\left(\tau_{-}\right)=$ $\infty$ it follows that the domain of definition of $\pi_{-}$is $[0, \infty)$ and $\lim _{a \rightarrow \infty} \pi_{-}(a)=\infty$.

Moreover when $\tau_{-} \in(0, \pi)$ we have

$b\left(\tau_{-}\right)-a\left(\tau_{-}\right)=\frac{2 \beta_{-}}{d_{1} \sin \tau_{-}}\left(\sinh \left(\gamma_{-} \tau_{-}\right)-\gamma_{-} \sin \tau_{-}\right)$.

Since $\sinh \left(\gamma_{-} \tau_{-}\right)>\gamma_{-} \sin \tau_{-}$when $\tau_{-} \in(0, \pi)$, we conclude from the expression above that $b\left(\tau_{-}\right)>$ $a\left(\tau_{-}\right)$if $\tau_{-} \in(0, \pi)$. Therefore $\pi_{-}(a)>a$ in $(0, \infty)$. So statement (a) is proved.

Derivating (6) with respect to $\tau_{-}$it follows that

$$
\begin{aligned}
\frac{d a}{d \tau_{-}} & =\frac{\beta_{-}}{d_{1} \sin ^{2} \tau_{-}} \varphi\left(\tau_{-},-\gamma_{-}\right) \quad \text { and } \\
\frac{d b}{d \tau_{-}} & =\frac{\beta_{-}}{d_{1} \sin ^{2} \tau_{-}} \varphi\left(\tau_{-}, \gamma_{-}\right) .
\end{aligned}
$$

Thus $\left(\pi_{-}\right)^{\prime}(a)=\frac{\varphi\left(\tau_{-}, \gamma_{-}\right)}{\varphi\left(\tau_{-},-\gamma_{-}\right)}=\frac{a}{b} e^{2 \gamma_{-} \tau_{-}}$and $\lim _{a \rightarrow 0}\left(\pi_{-}\right)^{\prime}(a)=1$. Therefore substatement (a.1) is proved.

Now we observe that

$$
\begin{gathered}
\left(\pi_{-}\right)^{\prime \prime}(a)=\frac{d}{d \tau_{-}}\left(\frac{d b}{d a}\right) \frac{1}{\frac{d a}{d \tau_{-}}}= \\
\frac{2 d_{1}\left(1+\gamma_{-}^{2}\right) \sin ^{3} \tau_{-}}{\beta_{-} \varphi\left(\tau_{-},-\gamma_{-}\right)^{3}}\left(\sinh \left(\gamma_{-} \tau_{-}\right)-\gamma_{-} \sin \tau_{-}\right)>0 .
\end{gathered}
$$

Therefore substatement (a.2) follows

From expression (6) it follows that

$$
\begin{aligned}
\lim _{a \rightarrow \infty} \frac{\pi_{-}(a)}{a} & =\lim _{\tau_{-} \rightarrow \pi} \frac{b\left(\tau_{-}\right)}{a\left(\tau_{-}\right)} \\
& =\lim _{\tau_{-} \rightarrow \pi} e^{2 \gamma_{-} \tau_{-}} \frac{\varphi\left(\tau_{-},-\gamma_{-}\right)}{\varphi\left(\tau_{-}, \gamma_{-}\right)}=e^{\gamma_{-} \pi} .
\end{aligned}
$$

Hence applying the L'Hôptal's rule we obtain substatement (a.3).

Since $t_{-}=0$ implies $\gamma_{-}=0$ it follows from (6) that $\pi_{-}(a)=a$ in $(0, \infty)$. As $\pi_{-}(0)=0$ we get that $\pi_{-}$is the identity. This proves statement (b).

Proposition 3.3. Consider the vector field $X_{i}$ in $R_{i}$ with $i \in\{-,+\}$ and with a virtual center or focus equilibrium and such that $t_{i}<0$. Let $\pi_{i}$ be the map associated to the Poincare map $\Pi_{i}: L_{i} \rightarrow L_{i}$ defined by the flow of the linear system $\dot{\mathbf{x}}=A_{i} \mathbf{x}+$ $B_{i}$. 
(a) The maps $\pi_{i}$ satisfy that $\pi_{i}:[0, \infty) \rightarrow[0, \infty)$, $\pi_{i}(0)=0, \lim _{a \rightarrow \infty} \pi_{i}(a)=+\infty$ and $\pi_{i}(a)<a$ in $(0, \infty)$.

(b) If $a \in(0, \infty)$ then $\left(\pi_{i}\right)^{\prime}(a)=\frac{a}{\pi_{i}(a)} e^{2 \gamma_{i} \tau_{i}}$. Moreover $0<\left(\pi_{i}\right)^{\prime}(a)<1$ and $\lim _{a \rightarrow 0}\left(\pi_{i}\right)^{\prime}(a)=$ 1.

(c) If $a \in(0, \infty)$ then $\left(\pi_{i}\right)^{\prime \prime}(a)<0$.

(d) The straight line $b=e^{\gamma_{i} \pi} a-t_{i}\left(1+e^{-\gamma_{i} \pi}\right) / d_{i}$ in the plane $(a, b)$ is an asymptote of the graph of $\pi_{i}$ when a tends to $+\infty$ where $\gamma_{i}=$ $t_{i} / \sqrt{4 d_{i}-t_{i}^{2}}$. So $\lim _{a \rightarrow \infty}\left(\pi_{1}\right)^{\prime}(a)=e^{\gamma_{i} \pi}$.

Proof. The proof follows in a similar way to the proof of Proposition 3.2.

Let $p_{-}$and $p_{+}$be the contact point of $\dot{\mathbf{x}}=$ $A_{o} \mathbf{x}+B_{o}$ with $L_{-}$and $L_{+}$respectively. We can define a Poincaré map $\Pi_{o}: D_{o}^{*} \subset L_{-} \rightarrow L_{+}$by $\Pi_{o}(q)=r$ being the map from points in $D_{o}$ to points in $L_{+}$defined by the flow of $\dot{\mathbf{x}}=A_{o} \mathbf{x}+B_{o}$ in forward time, where $D_{o}^{*}$ is a subset of $L_{-}$where the mapping $\Pi_{o}$ is well defined. So if $\varphi_{o}(s, q)$ is the solution of $\dot{\mathbf{x}}=A_{o} \mathbf{x}+B_{o}$ such that $\varphi_{o}(0, q)=q$ with $q \in D_{o}$, then $r=\varphi_{o}(s, q), s>0$ with $r \in L_{+}$.

As before we can see the mapping $\Pi_{o}$ in a different way. Given, $q \in D_{o}^{*}$ and $r \in L_{+}$there exist unique $b \geq 0$ and $c \geq 0$ such that $q=p_{-}+b \dot{p}_{-}$and $r=p_{+}-c \dot{p}_{+}$, where $\dot{p}_{+}=X_{+}\left(p_{+}\right)=\left(0, b_{2}+1\right)$. So the mapping $\Pi_{o}$ induces a mapping $\pi_{o}$ given by $\pi_{o}(b)=c$. To study the qualitative behavior of $\Pi_{o}$ is equivalent to study the qualitative behavior of $\pi_{o}$. As before we will consider the map $\pi_{o}$ instead of $\Pi_{o}$.

In the same way we can define a first return map $\bar{\Pi}_{o}: \bar{D}_{o}^{*} \subset L_{+} \rightarrow L_{-}$and the respective $\bar{\pi}_{o}$.

The next propositions state the qualitative behavior of these maps.

Proposition 3.4. Consider the vector field $X_{o}$ in $R_{o}$ with $t_{o}>0$. Let $\pi_{o}$ be the map associated to the Poincaré map $\Pi_{o}: D_{o}^{*} \subset L_{-} \rightarrow L_{+}$defined by the flow of the linear system $\dot{\mathbf{x}}=A_{o} \mathbf{x}+B_{o}$ from the straight line $L_{-}$to the straight line $L_{+}$.

(a) If $0<b_{2}<1$ then the map $\pi_{o}$ satisfies that $\pi_{o}:\left[b^{*}, \infty\right) \rightarrow\left[c^{*}, \infty\right), b^{*}, c^{*} \geq 0$ with $\pi_{o}\left(b^{*}\right)=c^{*}$ and $\lim _{b \rightarrow \infty} \pi_{o}(b)=+\infty$. Moreover $b^{*}=0$ if and only if $e^{\gamma_{0} \pi} \geq \frac{1+b_{2}}{1-b_{2}}$ and $c^{*}=0$ if and only if $e^{\gamma_{0} \pi} \leq \frac{1+b_{2}}{1-b_{2}}$.

(a.1) If $b \in\left(b^{*}, \infty\right)$ then $\pi_{o}^{\prime}(b)=$ $\left(\frac{1-b_{2}}{1+b_{2}}\right)^{2} e^{2 \gamma_{o} \tau_{o}} \frac{b}{\pi_{o}(b)}$, with $\tau_{o} \rightarrow 0$ when $b \rightarrow \infty$ and $\lim _{b \rightarrow \infty} \pi_{o}^{\prime}(b)=\left(\frac{1-b_{2}}{1+b_{2}}\right)^{2}$.

(b) If $-1<b_{2} \leq 0$ then $b \in(0, \infty)$ and $\pi_{o}$ satisfies $\pi_{o}:[0, \infty) \rightarrow\left[c^{*}, \infty\right), c^{*}>0$ with $\pi_{o}(0)=c^{*}$ and $\lim _{b \rightarrow \infty} \pi_{o}(b)=+\infty$.

(b.1) $\pi_{o}^{\prime}(b)$ satisfy the same conditions described in (a.1).

Proof. The solution of $\dot{\mathbf{x}}=A_{o} \mathbf{x}+B_{o}$ that pass through $q=p_{-}+b \dot{p}_{-}$is $\left(x\left(\tau_{o}\right), y\left(\tau_{o}\right)\right)$ given by

$$
\left(\begin{array}{c}
e^{\gamma_{o} \tau_{o}}\left(\left(b_{2}-1\right) \cos \tau_{o}+\frac{2\left(1-b_{2}\right)\left(2 b+a_{1}\right)}{\beta_{o}} \sin \tau_{o}\right)-b_{2} \\
-\frac{e^{\gamma_{o} \tau_{o}}}{2}\left(2 b\left(1-b_{1}\right) \cos \tau_{o}+\frac{\left(1-b_{2}\right)\left(4 b a_{1}+8\right)}{\beta_{o}} \sin \tau_{o}\right)
\end{array}\right),
$$

where $\alpha_{o}=\frac{t_{o}}{2}, \beta_{o}=\frac{\sqrt{4-a_{1}^{2}}}{2}, \tau_{o}=\beta_{o} s$ and $\gamma_{o}=$ $\frac{\alpha_{o}}{\beta_{o}}$.

In order that the mapping $\pi_{o}$ be defined in 0 and $\pi_{o}(0)=0$, we must have $e^{\gamma_{o} \pi}=\frac{1+b_{2}}{1-b_{2}}$. Moreover if $e^{\gamma_{o} \pi}>\frac{1+b_{2}}{1-b_{2}}$, then $\pi_{o}$ is also defined in 0 and $\pi_{o}(0)=c^{*}>0$. On the other hand if $e^{\gamma_{o} \pi}<\frac{1+b_{2}}{1-b_{2}}$ then $\pi_{o}$ is defined in the interval $\left[b^{*}, \infty\right)$, where $b^{*}>0$ and $\pi_{o}\left(b^{*}\right)=0$.

In the domain of definition of $\pi_{o}$ we obtain from expression (7) the parametric solution of $c=\pi_{o}(b)$ given by

$$
b\left(\tau_{o}\right)=-\alpha_{o}+\frac{\beta_{o} e^{-\gamma_{o} \tau_{o}}}{\sin \tau_{o}}\left(\frac{1+b_{2}}{1-b_{2}}+e^{\gamma_{o} \tau_{o}} \cos \tau_{o}\right)
$$

and

$$
c\left(\tau_{o}\right)=\alpha_{o}+\frac{\beta_{o} e^{\gamma_{o} \tau_{o}}}{\sin \tau_{o}}\left(\frac{1-b_{2}}{1+b_{2}}+e^{-\gamma_{o} \tau_{o}} \cos \tau_{o}\right) .
$$

Using the same arguments than in Proposition 3.2 we can prove the statements (a) and (a.1).

Statement (b) can be obtained in the same way. 
Proposition 3.5. Consider the vector field $X_{o}$ in $R_{o}$ with $t_{o}<0$. Let $\pi_{o}$ be the map associated to the Poincaré map $\Pi_{o}: D_{o}^{*} \subset L_{-} \rightarrow L_{+}$defined by the flow of the linear system $\dot{\mathbf{x}}=A_{o} \mathbf{x}+B_{o}$ from the straight line $L_{-}$to the straight line $L_{+}$.

(a) If $-1<b_{2}<0$ then $\pi_{o}$ satisfies that $\pi_{o}$ : $\left[b^{*}, \infty\right) \rightarrow\left[c^{*}, \infty\right), b^{*}, c^{*} \geq 0$ with $\pi_{o}\left(b^{*}\right)=c^{*}$ and $\lim _{b \rightarrow \infty} \pi_{o}(b)=+\infty$. Moreover $b^{*}=0$ if and only if $e^{\gamma_{o} \pi} \geq \frac{1+b_{2}}{1-b_{2}}$ and $c^{*}=0$ if and only if $e^{\gamma_{o} \pi} \leq \frac{1+b_{2}}{1-b_{2}}$.

(a.1) If $b \in\left(b^{*}, \infty\right)$ then $\pi_{o}^{\prime}(b)=$ $\left(\frac{1-b_{2}}{1+b_{2}}\right)^{2} e^{2 \gamma_{o} \tau_{o}} \frac{b}{\pi_{o}(b)}$ with $\tau_{o} \rightarrow 0$ when $b \rightarrow \infty$ and $\lim _{b \rightarrow 0} \pi_{o}^{\prime}(b)=\infty$ and $\lim _{b \rightarrow \infty} \pi_{o}^{\prime}(b)=\left(\frac{1-b_{2}}{1+b_{2}}\right)^{2}$.

(b) If $0 \leq b_{2}<1$ then $\pi_{o}$ satisfies $\pi_{o}$ : $\left[b^{*}, \infty\right) \rightarrow[0, \infty), b^{*}>0$ with $\pi_{o}\left(b^{*}\right)=0$ and $\lim _{b \rightarrow \infty} \pi_{o}(b)=+\infty$.

(b.1) $\pi_{o}^{\prime}(b)$ satisfies the same conditions described in (a.1).

Proof. The proof follows using the same ideas of the previous proposition and will be omitted.

Proposition 3.6. Consider the vector field $X_{o}$ in $R_{o}$ with $t_{o}>0$. Let $\bar{\pi}_{o}$ be the map associated to the Poincaré map $\bar{\Pi}_{o}: \bar{D}_{o}^{*} \subset L_{+} \rightarrow L_{-}$defined by the flow of $X_{o}$ from straight line $L_{+}$to the straight line $L_{-}$.

(a) If $-1<b_{2}<0$ then $\bar{\pi}_{o}$ satisfies that $\bar{\pi}_{o}$ : $\left[d^{*}, \infty\right) \rightarrow\left[a^{*}, \infty\right), d^{*}, a^{*} \geq 0$ with $\bar{\pi}_{o}\left(d^{*}\right)=$ $a^{*}$ and $\lim _{d \rightarrow \infty} \bar{\pi}_{o}(d)=+\infty$. Moreover $d^{*}=0$ if and only if $e^{\gamma_{o} \pi} \geq \frac{1-b_{2}}{1+b_{2}}$ and $a^{*}=0$ if and only if $e^{\gamma_{o} \pi} \leq \frac{1-b_{2}}{1+b_{2}}$.

(a.1) If $d \in\left(d^{*}, \infty\right)$ then $\bar{\pi}_{o}^{\prime}(d)=$ $\left(\frac{1+b_{2}}{1-b_{2}}\right)^{2} e^{2 \gamma_{o} \bar{\tau}_{o}} \frac{d}{\bar{\pi}_{o}(d)}$ with $\bar{\tau}_{o} \rightarrow 0$ when $d \rightarrow \infty$ and $\lim _{d \rightarrow 0} \bar{\pi}_{o}^{\prime}(d)=\infty$ and $\lim _{d \rightarrow \infty} \bar{\pi}_{o}^{\prime}(d)=\left(\frac{1+b_{2}}{1-b_{2}}\right)^{2}$. (b) If $0 \leq b_{2}<1$ then $d \in(0, \infty)$ and $\bar{\pi}_{o}$ satisfies $\bar{\pi}_{o}:[0, \infty) \rightarrow\left[a^{*}, \infty\right), a^{*}>0$ with $\bar{\pi}_{o}(0)=a^{*}$ and $\lim _{d \rightarrow \infty} \bar{\pi}_{o}(d)=+\infty$.

(b.1) $\bar{\pi}_{o}^{\prime}(d)$ satisfy the same conditions described in (a.1).

Proposition 3.7. Consider the vector field $X_{o}$ in $R_{o}$ with $t_{o}<0$. Let $\bar{\pi}_{o}$ be the map associated to the Poincaré map $\bar{\Pi}_{o}: \bar{D}_{o}^{*} \subset L_{+} \rightarrow L_{-}$defined by the flow of $X_{o}$ from the straight line $L_{+}$to the straight line $L_{-}$.

(a) If $0 \leq b_{2}<1$ then $\bar{\pi}_{o}$ satisfies that $\bar{\pi}_{o}$ : $\left[d^{*}, \infty\right) \rightarrow\left[a^{*}, \infty\right), d^{*}, a^{*} \geq 0$ with $\bar{\pi}_{o}\left(d^{*}\right)=$ $a^{*}$ and $\lim _{d \rightarrow \infty} \bar{\pi}_{o}(a)=+\infty$. Moreover $d^{*}=0$ if and only if $e^{\gamma_{o} \pi} \geq \frac{1-b_{2}}{1+b_{2}}$ and $a^{*}=0$ if and only if $e^{\gamma_{o} \pi} \leq \frac{1-b_{2}}{1+b_{2}}$.

(a.1) If $d \in\left(d^{*}, \infty\right)$ then $\bar{\pi}_{o}^{\prime}(d)=$ $\left(\frac{1+b_{2}}{1-b_{2}}\right)^{2} e^{2 \gamma_{o} \bar{\tau}_{o}} \frac{b}{\pi_{o}(b)}$ with $\tau_{o} \rightarrow 0$ when $d \rightarrow \infty$ and $\lim _{d \rightarrow 0} \bar{\pi}_{o}^{\prime}(d)=\infty$ and $\lim _{d \rightarrow \infty} \bar{\pi}_{o}^{\prime}(d)=\left(\frac{1+b_{2}}{1-b_{2}}\right)^{2}$.

(b) If $-1<b_{2}<0$ then $d \in(0, \infty)$ and $\bar{\pi}_{o}$ satisfies $\bar{\pi}_{o}:[0, \infty) \rightarrow\left[a^{*}, \infty\right), a^{*}>0$ with $\bar{\pi}_{o}(0)=a^{*}$ and $\lim _{d \rightarrow \infty} \bar{\pi}_{o}(d)=+\infty$.

(b.1) $\bar{\pi}_{o}^{\prime}(d)$ satisfy the same conditions described in (a.1).

\section{Limit Cycles having a Focus in $R_{O}$}

In what follows without loss of generality we will suppose that the center of hypothesis (H2) is in $R_{-}$.

Proposition 4.1. Assume that system (3) satisfies assumptions (H1) and (H2). Suppose that the real equilibrium point in $R_{O}$ is between $L_{-}$and $L_{o}$ where $L_{o}$ is a line parallel to $L_{+}$through the origin. Then there exists a unique limit cycle of (3), which is hyperbolic. Moreover this limit cycle visits the three regions $R_{-}, R_{o}$ and $R_{+}$. It is a repeller if $t_{o}<0$, and an attractor if $t_{o}>0$. 
Proof. Suppose that we have a center in $X_{-}$, and a focus in $X_{o}$ and in $X_{+}$. By the hypotheses we have $0 \leq b_{2}<1$.

Using the previous notation we have $\gamma_{i}=$ $\frac{\alpha_{i}}{\beta_{i}}, i \in\{-, o,+\}$. So $\gamma_{-}=0$ and $\gamma_{o}, \gamma_{+} \neq 0$. The domain of the first return map $\Pi$ defined by

$$
\Pi=\bar{\pi}_{o} \circ \pi_{+} \circ \pi_{o} \circ \pi_{-}
$$

is an interval of $\mathbb{R}^{+}$that depends on the domain of the mapping $\pi_{o}$ and $\bar{\pi}_{o}$ stated in Propositions 3.4, $3.5,3.6$ and 3.7 .

Suppose that $\gamma_{o}>0$ and $\gamma_{+}<0$. In this case $\bar{D}_{o}\left(\bar{\pi}_{o}\right)=[0, \infty)$ and $D_{o}\left(\pi_{o}\right)=\left[b^{*}, \infty\right)$ where $b^{*} \geq 0$ and $\pi_{o}\left(b^{*}\right)=0$ (see figure 3 ). This implies that $D(\Pi)=\left[a^{*}, \infty\right)$ where $a^{*}=b^{*}$. Moreover we have $\Pi:\left[a^{*}, \infty\right) \rightarrow\left[a^{* *}, \infty\right)$ with $a^{* *}>a^{*}$ and $\Pi\left(a^{*}\right)=$ $a^{* *}$.

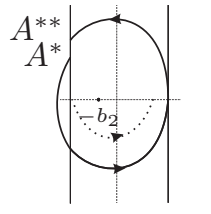

$\Pi\left(a^{*}\right)=a^{* *}$

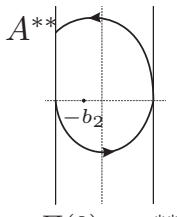

$\Pi(0)=a^{* *}$

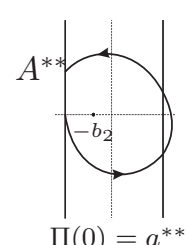

$\Pi(0)=a^{* *}$
Fig. 3. The flow of the three zones vector field with the real equilibrium between $L_{-}$and $L_{o}$ where $A^{*}=$ $p_{-}-a^{*} X_{-}\left(p_{-}\right)$and $A^{* *}=p_{-}-a^{* *} X_{-}\left(p_{-}\right)$.

Define the displacement function

$$
h(a)=\Pi(a)-a .
$$

Note that finding a fixed point of $\Pi$ is equivalent to find zeroes of the function $h$.

In $a^{*}$ we have $h\left(a^{*}\right)=\Pi\left(a^{*}\right)-a^{*}>0$. Supposing that $h$ admits a zero and that $a_{s}$ is the smallest zero we must have $h^{\prime}\left(a_{s}\right) \leq 0$, or equivalently $\Pi^{\prime}\left(a_{s}\right) \leq 1$. But from the definition of $\Pi$ we can write

$$
\Pi^{\prime}(a)=\bar{\pi}_{o}^{\prime}(d) \pi_{+}^{\prime}(c) \pi_{o}^{\prime}(b) \pi_{-}^{\prime}(a)
$$

where $b=\pi_{-}(a)=a, c=\pi_{o}(b)$ and $d=\bar{\pi}_{o}(c)$.

From Propositions 3.2, 3.3, 3.4, 3.5, 3.6 and 3.7 it follows that

$$
\begin{aligned}
& \Pi^{\prime}(a)= \\
& \left(\frac{1+b_{2}}{1-b_{2}}\right)^{2} \frac{d}{\bar{\pi}_{o}(d)} e^{2 \gamma_{o} \bar{\tau}_{o}} \frac{c}{\pi_{+}(c)} e^{2 \gamma_{+} \tau_{+}}\left(\frac{1-b_{2}}{1+b_{2}}\right)^{2} \frac{b}{\pi_{o}(b)} e^{2 \gamma_{o} \tau_{o}} \\
& =\frac{a}{\Pi(a)} e^{2\left(\gamma_{o}\left(\tau_{o}+\bar{\tau}_{o}\right)+\gamma_{+} \tau_{+}\right)},
\end{aligned}
$$

with $\tau_{+} \in(0, \pi)$ increasing with $a$, and $\bar{\tau}_{o}+\tau_{o} \in$ $\left(0,2 \pi-\tau^{*}\right)$ decreasing with $a$. So $\gamma_{o}\left(\tau_{o}+\bar{\tau}_{o}\right)+\gamma_{+} \tau_{+}$is a decreasing function in $a$ and $\gamma_{o}\left(\tau_{o}+\bar{\tau}_{o}\right)+\gamma_{+} \tau_{+} \rightarrow$ $\gamma_{+} \pi<0$ when $a \rightarrow \infty$. Hence from (8) and from the fact that $\Pi^{\prime}\left(a_{s}\right) \leq 1$ we must have

$$
\gamma_{o}\left(\tau_{o s}+\bar{\tau}_{o s}\right)+\gamma_{+} \tau_{+s} \leq 0 .
$$

Now supposing that $\Pi$ admits a second fixed point $a_{r}$ from the monotonicity of the function $\gamma_{o}\left(\tau_{o}+\right.$ $\left.\bar{\tau}_{o}\right)+\gamma_{+} \tau_{+}$we must have

$\gamma_{o}\left(\tau_{o s}+\bar{\tau}_{o s}\right)+\gamma_{+} \tau_{+s}=0$ and $\gamma_{o}\left(\tau_{o r}+\bar{\tau}_{o r}\right)+\gamma_{+} \tau_{+r}<0$.

As $a_{s}$ and $a_{r}$ are fixed point of $\Pi$ this implies from (8) that $\Pi^{\prime}\left(a_{s}\right)=1$ and $\Pi^{\prime}\left(a_{r}\right)<1$. So in this case, for $a \in\left(a_{s}, a_{r}\right)$ we obtain $\Pi(a)>a$. Now from (8) it follows that $\Pi^{\prime}(a)<1$ for $a \in\left(a_{s}, a_{r}\right)$ and from the Mean Value Theorem we have

$$
\Pi(a)-\Pi\left(a_{s}\right)=\Pi^{\prime}(\bar{a})\left(a-a_{s}\right)<a-a_{s}
$$

that implies that $\Pi(a)<a$. This is a contradiction and so we have at most a fixed point $a_{s}$ for $\Pi$ and

$$
\gamma_{o}\left(\tau_{o s}+\bar{\tau}_{o s}\right)+\gamma_{+} \tau_{+s}<0
$$

On the other hand since

$$
h^{\prime}(a)=\Pi^{\prime}(a)-1 \text { and } \lim _{a \rightarrow \infty} h^{\prime}(a)=e^{2 \gamma_{+} \pi}-1<0,
$$

it follows by the Mean Value Theorem that $\lim _{a \rightarrow \infty} h(a)=-\infty$, and this shows that $h$ admits a $a \rightarrow \infty$
zero. Moreover this zero is equivalent to a fixed point of the first return map $\Pi$ and this fixed point is a hyperbolic attractor because from (9) we have $\Pi^{\prime}\left(a_{s}\right)<1$.

Now suppose that $\gamma_{o}<0$ and $\gamma_{+}>0$. In this case we can use the same idea of the previous case and define a first return map $\Pi:\left[a^{*}, \infty\right) \rightarrow\left[a^{* *}, \infty\right)$ where $\Pi\left(a^{*}\right)=a^{* *}$ and $a^{* *}<a^{*}$. But now we obtain a unique limit cycle that is a hyperbolic repeller.

Now from Proposition 15 of [Freire et al., 1998] it is not possible to have a limit cycle that visit only the regions $R_{o}$ and $R_{+}$otherwise we would have two hyperbolic attractor limit cycle containing the same equilibrium point what is not possible.

This finish the prove of the theorem. 
Proposition 4.2. Assume that system (3) satisfies assumptions (H1) and (H2). Suppose that the real equilibrium point in $R_{o}$ is between $L_{o}$ and $L_{+}$. Then there exists a unique limit cycle of (3), which is hyperbolic. This limit cycle visits the three regions $R_{-}, R_{o}$ and $R_{+}$if $D(\Pi)=[0, \infty)$ and $\Pi(0)>0$ and visits only the regions $R_{o}$ and $R_{+}$otherwise.

Proof. Suppose that $\gamma_{o}>0$ and $\gamma_{+}<0$. We have $-1<b_{2}<0$. In this case the real focus of $X_{o}$ is a repeller and the virtual focus of $X_{+}$is an attractor.

$$
\text { If } e^{\gamma_{o} \pi} \geq \frac{1-b_{2}}{1+b_{2}} \text {, then } \bar{D}_{o}\left(\bar{\pi}_{o}\right)=[0, \infty) \text { and }
$$
$D_{o}\left(\pi_{o}\right)=[0, \infty)$ (see figure 4). This implies that $D(\Pi)=[0, \infty)$ and by the fact that the real focus is a repeller it is not difficult to see that $\Pi(0)=$ $a^{* *}>0$. In this scenario a similar analysis as the one done in the proof of Proposition 4.1 shows that there exists a unique limit cycle that visits the three regions, and which is hyperbolic.
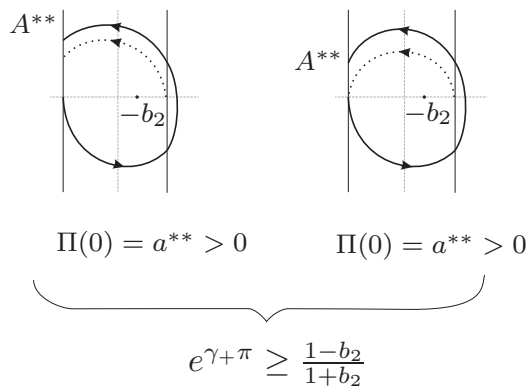

Fig. 4. The flow of the three zones vector field with the real equilibrium between $L_{o}$ and $L_{+}$where $A^{* *}=p_{-}-a^{* *} X_{-}\left(p_{-}\right)$.

On the other hand, if $e^{\gamma_{0} \pi}<\frac{1-b_{2}}{1+b_{2}}$, then $D\left(\bar{\pi}_{o}\right)=\left[d^{*}, \infty\right)$ with $d^{*}>0, D\left(\pi_{o}\right)=[0, \infty)$ with $\pi_{o}(0)=c^{*}>0$. Here three cases are possible.

Case 1: $\pi_{+}\left(c^{*}\right)<d^{*}$. In this case $D(\Pi)=\left[a^{*}, \infty\right)$ where $a^{*}>0$ is such that $\left(\pi_{+} \circ \pi_{o}\right)\left(a^{*}\right)=d^{*}$ and so $\Pi\left(a^{*}\right)=0<a^{*}$ (see figure $5(1)$ ). From this figure as the real equilibrium point is repeller it follows that there is a stable limit cycle that visits the regions $R_{o}$ and $R_{+}$. We observe that for this limit cycle we can use the Proposition 15 of [Freire et al., 1998] and conclude that it is hyperbolic. We can also define a restricted Poincaré return map $\Pi_{r}$ near this hyperbolic stable limit cycle and we obtain $\Pi_{r}^{\prime}(\bar{a})=e^{2\left(\gamma_{o} \tau_{o}^{r}+\gamma_{+} \tau_{+}^{r}\right)}<1$ where $\bar{a}$ is the point associated to the limit cycle, for more de- tails see equation (31) of [Freire et al., 1998]. This implies that $\gamma_{o} \tau_{o}^{r}+\gamma_{+} \tau_{+}^{r}<0$. Now in the points of the domain of definition of the Poincaré return map $\Pi$ it is easy to see that $\tau_{o}+\bar{\tau}_{o}<\tau_{o}^{r}$ and $\tau_{+}>\tau_{+}^{r}$, and as $\gamma_{o}>0$ and $\gamma_{+}<0$ we obtain $\gamma_{o}\left(\tau_{o}+\bar{\tau}_{o}\right)+\gamma_{+} \tau_{+}<0$. Now if $\Pi$ admits a fixed point and $a_{s}$ is the smallest fixed point it follows from the fact that $\Pi\left(a^{*}\right)=0<a^{*}$ that we must have $\Pi\left(a_{s}\right)=a_{s}$ and $\Pi^{\prime}\left(a_{s}\right) \geq 1$. But from equation (8) we conclude that $\Pi^{\prime}\left(a_{s}\right)=e^{2\left(\gamma_{o}\left(\tau_{o s}+\bar{\tau}_{o s}\right)+\gamma_{+} \tau_{+s}\right)}<1$, because $\gamma_{o}\left(\tau_{o s}+\bar{\tau}_{o s}\right)+\gamma_{+} \tau_{+s}<0$. This contradiction implies that a such fixed point $a_{s}$ does not exist.

Case 2: $\pi_{+}\left(c^{*}\right)=d^{*}$ In this case we have a limit cycle tangent to $L_{-}$because $\Pi(0)=0$ (see figure 5 (2)). From Proposition 15 of [Freire et al., 1998] this limit cycle is hyperbolic attractor. This implies that $\Pi^{\prime}(0)<1$. Now as the previous case we cannot have a fixed point for $\Pi$ because again if $a_{s}$ is the smallest fixed point of $\Pi$ we must have $\Pi^{\prime}\left(a_{s}\right) \geq 1$ and this is a contradiction as in case 1 . So in this case only one limit cycle exists, it is hyperbolic and it visits only the regions $R_{o}$ and $R_{+}$.

Case 3: $\pi_{+}\left(c^{*}\right)>d^{*}$ In this case $D(\Pi)=[0, \infty)$ and $\Pi(0)=a^{* *}>0$ (see figure 5(3)) and a similar argument as the one given in the proof of Proposition 4.1 shows that there exists a unique limit cycle, which is hyperbolic and it visits the three regions.

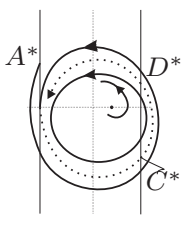

(1) $\Pi\left(a^{*}\right)=0$

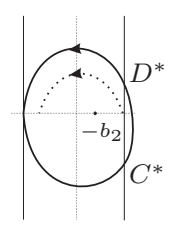

(3) $\Pi(0)=0$

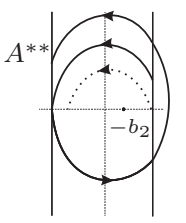

(2) $\Pi(0)=a^{* *}$
Fig. 5. Three possible cases for the flow of the three zones vector field when the real equilibrium is between $L_{o}$ and $L_{+}$and $e^{\gamma_{+} \pi}<\frac{1-b_{2}}{1+b_{2}}$. $A^{*}=$ $p_{-}-a^{*} X_{-}\left(p_{-}\right), C^{*}=p_{+}-c^{*} X_{+}\left(p_{+}\right), D^{*}=$ $p_{+}+d^{*} X_{+}\left(p_{+}\right)$and $A^{* *}=p_{-}-a^{* *} X_{-}\left(p_{-}\right)$.

The situation when $\gamma_{o}<0$ and $\gamma_{+}>0$ can be analyzed in the same way that in the previous case and the details are omitted.

Proof of Theorem 1.1. The proof of Theorem 1.1 follows directly from the proof of Propositions 4.1 and 4.2 . 
We note that the existence of the hyperbolic limit cycle of Theorem 1.1 does not depend on the sign of $\gamma_{o}+\gamma_{+}$as the result given in Proposition 15 of [Freire et al., 1998].

\section{Acknowledgments}

The first author is partially supported by $\mathrm{CNPq}$ grand number 200293/2010-9. The second author is partially supported by MCYT/FEDER grant MTM2008-03437, CIRIT grant number 2009SGR410 and by ICREA Academia. Both authors are also supported by the joint project CAPES-MECD grant PHB-2009-0025-PC.

\section{References}

Andronov, A., Vit, A., \& Khaikin, S., [1966] Theory of Oscillators, Dover.

Chua, L.O., \& Lin, G., [1990] "Canonical realization of Chua's circuit family," IEEE Trans. Circuits Syst. CAS no 7, 37, 885-902.

Dumortier, F., Llibre, J., \& Artés, J.C., [2006] Qualitative theory of planar differential systems, Universitext, Springer-Verlag, New York.

FitzHugh, R., [1961] "Impulses and physiological states in theoretical models of nerve membrane," Biophys. J. 1, 445-466.

Lefschetz, S., [1965] Stability of Nonlinear Control Systems Academic Press, New York.

Llibre, J., \& Sotomayor, J., [1996] "Phase portaits of planar control systems," Nonlin. Anal. Th. Meth. Appl. 27, 1177-1097.

Llibre, J., \& Teruel, E., [2009] "Qualitative theory of planar piecewise linear differential systems," App. Math. and Computation 215, 140-146.

Llibre, J., \& Teruel, E., [2004] "Existence of Poincaré Maps in piecewise linear differential systems in $\mathbb{R}^{n}$," Int. J. of Bif. and Chaos. 14, 2843-2851.

Nagumo, J.S., Arimoto, S., \& Yoshizawa, S., [1962] "An active pulse transmission line simulating nerve axon," Proc. IRE 50, 2061-2071.
Narendra, K.S., \& Taylor, J.M., [1973] Frequency Domain Criteria for Absolute Stability Academic Press, New York.

Freire, E., Ponce, E., Rodrigo, F., \& Torres, F., [1998] "Bifurcation sets of continuous piecewise linear systems with two zones," Int. J. of Bif. and Chaos 11, 2073-2097. 